\title{
The SEED study of dorzagliatin in drug-naiive patients with type 2 diabetes: a randomized, double- blind, placebo-controlled, phase 3 trial
}

\section{Li Chen ( $\square$ lichen@huamedicine.com )}

Hua Medicine (Shanghai) Ltd. https://orcid.org/0000-0002-6192-3752

\section{Dalong Zhu}

Nanjing University Medical School Affiliated Nanjing Drum Tower Hospital

\section{Xiaoying Li}

Department of Endocrinology and Metabolism, Fudan Institute of Metabolic Diseases, Zhongshan

Hospital, Fudan University, Shanghai 200032

\section{Wenying Yang}

China-Japan Friendship Hospital

Jianhua Ma

Nanjing First Hospital

Jiao'e Zeng

Jingzhou Central Hospital

\section{Shenglian Gan}

The First People's Hospital of Changde City

\section{Xiaolin Dong}

Jinan Central Hospital Affiliated to Shandong University

\section{Jing Yang}

Department of Endocrinology, The First Hospital of Shanxi Medical University

\section{Xiaohong Lin}

ZHUZHOU CENTRAL HOSPITAL

\section{Hanqing Cai}

Jilin University Second Hospital

\section{Weihong Song}

Chenzhou No 1 People's Hospital

\section{Xuefeng Li}

Taihe Hospital

\section{Keqin Zhang}

Tongji Hospital Affiliated to Tongji University

\section{Qiu Zhang}

First Affiliated Hospital of Anhui Medical University 


\section{Yibing Lu}

Nanjing Medical University Second Affiliated Hospital

\section{Ruifang Bu}

WUXI PEOPLE'S HOSPITAL

\section{Huige Shao}

CHANGSHA CENTRAL HOSPITAL

\section{Guixia Wang}

Department of Endocrinology, The First Hospital of Jilin University

\section{Guoyue Yuan}

Affiliated Hospital of Jiangsu University

\section{Xingwu Ran}

West China Hospital, Sichuan University, Endocrinology and Metabolism

\section{Lin Liao}

Shandong Provincial Qianfoshan Hospital

\section{Wenjuan Zhao}

The Affiliated Hospital of Qingdao University

\section{Li Sun}

Si Ping Centre People's Hospital

\section{Lixin Shi}

The First Hospital of Guiyang Medical School, Endocrinology

\section{Zhaoshun Jiang}

PLA joint logistic support force 960 hospital

\section{Yaoming Xue}

Southern Medical University Nanfang Hospital

\section{Hongwei Jiang}

Henan University of Science and Technology Affiliated First Hospital

\section{Quanmin Li}

General Hospital of PLA Second Artillery, The department of Endocrinology and metabolism

\section{Zongbao Li}

Hainan N0.3 Provincial People's Hospital

\section{Maoxiong Fu}

The Second Affiliated Hospital of Hainan Medical University

\section{Zerong Liang}

Chongqing Red Cross Hospital

\section{Lian Guo}

Chongqing Three Gorges Central Hospital, Department of Endocrinology

\section{Ming Liu}

Tianjin Medical University General Hospital

\section{Chun Xu}


The third medical center of PLA general hospital

\section{Wenhui Li}

Peking Union Medical College Hospital, Department of Endocrinology

\section{Xuefeng Yu}

Tongji Hospital of Tongji Medical College of Huazhong University of Science and Technology

\section{Guijun Qin}

Zhengzhou University

\section{Zhou Yang}

JiangXi PingXiang People's Hospital

\section{Benli Su}

The Second Hospital of Dalian Medical University

\section{Longyi Zeng}

Third Affiliated Hospital of Sun Yat-Sen University

\section{Houfa Geng}

Xuzhou Central Hospital

\section{Yongquan Shi}

Shanghai Changzheng Hospital

\section{Yu Zhao}

Hua Medicine (Shanghai) Ltd https://orcid.org/0000-0001-5245-4379

\section{Yi Zhang}

Hua Medicine (Shanghai) Ltd

\section{Article}

Keywords: SEED study, diabetes, glucose, insulin

Posted Date: July 22nd, 2021

DOI: https://doi.org/10.21203/rs.3.rs-733611/v1

License: (9) (1) This work is licensed under a Creative Commons Attribution 4.0 International License. Read Full License

Version of Record: A version of this preprint was published at Nature Medicine on May 12th, 2022. See the published version at https://doi.org/10.1038/s41591-022-01802-6. 


\section{Abstract}

Improvement of glucose and insulin sensitivity remains an unmet medical need in diabetes management, in which the underlying cause of type 2 diabetes(T2D) was not well addressed by current treatment. We report the findings of a randomized, double-blind, placebo-controlled, phase 3 clinical trial (NCT03173391) to evaluate the efficacy and safety of dorzagliatin, a dual-acting glucokinase (GK) allosteric modulator, which enhances the GK activity in T2D patients in a glucose dependent manner, and has demonstrated its effects in blood glucose control through improvement of glucose sensitivity in T2D patients. Eligible drug-naïve T2D patients $(n=463)$ were randomly assigned to dorzagliatin group or placebo group in a 2:1 ratio for a 24-week double-blind treatment, then followed by a 28-week open-label treatment with dorzagliatin in all patients. The primary efficacy endpoint was the change from baseline in the glycated hemoglobin (HbA1c) level at week 24. Safety was assessed throughout the trial. At week 24, the change from baseline in $\mathrm{HbA} 1 \mathrm{c}$ was $-1.07 \%$ in the dorzagliatin group and $-0.50 \%$ with placebo (ETD, $-0.57 \% ; 95 \% \mathrm{Cl},-0.79$ to $-0.36 ; \mathrm{P}<0.001)$, and the effects sustained through 52 weeks. Improvement of $\beta-$ cell function was demonstrated by an increase of HOMA2- $\beta$ in dorzagliatin group over placebo group ( 2.56 vs $-0.72 ; 95 \% \mathrm{Cl}, 0.44$ to $6.11 ; \mathrm{P}<0.05)$ at week 24 . The incidence of adverse events(AEs) was similar between the two groups during the 24 weeks and most AEs were mild during 52 weeks. There were no severe hypoglycemia events and drug related serious adverse events. The incidence of hypoglycemia was 1 of 310 patients $(0.3 \%)$ in the dorzagliatin group during the 24 weeks. There was no body weight gain during the study period. In drug-naïve T2D patients, dorzagliatin demonstrated fast onset and sustained glycemic control with a good safety and tolerability profile for 52 weeks.

\section{Introduction}

Type 2 diabetes is a complex, chronic metabolic disorder, characterized by insulin resistance and a progressive loss of $\beta$-cell function, which requires continuous medical care with various interventions to control the blood glucose in order to reduce diabetes complications. ${ }^{1}$ Current therapies have not been able to treat the underlying cause of diabetes and stop the progression of the disease, which encourage the medical community to seek for disease-modifying therapy. ${ }^{2}$ The loss of glucokinase function in diabetes leads to a reduced glucose sensitivity in glucose stimulated insulin release(GSIR) that results in a delayed insulin secretion. The impaired early phase insulin secretion generates a mismatch of glucose and insulin signals in glucose storage in the liver and muscle which eventually leads to a dysfunction of glucose homeostasis. ${ }^{3}$ It is highly desirable to develop a therapeutics that directly optimizes the glucose sensor function of glucokinase and synchronizes the glucose signal with glucose-controlling hormones such as insulin, glucagon and glucagon-like peptide-1, to achieve improved glucose and insulin sensitivity in diabetes patients.

Glucokinase(GK), is the glucose sensor which responds to the glucose fluctuation to regulate glucose storage and production $\cdot{ }^{4-7}$ Glucokinase is mainly expressed in glucose-sensitive tissues, including pancreas, liver and intestine etc. In pancreas, it directly controls the set point of blood glucose and the 
threshold of glucose-induced hormone secretion, including glucose-dependent regulation of early-phase insulin secretion, glucagon and glucagon-like peptide-1(GLP-1) release, in response to the glucose level during feeding and fasting. The majority of GK is found in hepatocytes, which is tightly regulated by glucokinase regulatory protein (GKRP) in response to hepatic glucose change, and controls the hepatic glucose uptake and glycogen synthesis. Genetic defects in glucokinase cause the dysregulation of insulin release, clinical hyperinsulinemia(HI) and Maturity Onset Diabetes of the Young-2(MODY-2). ${ }^{8}$ Significant reduction of insulin secretion and hepatic glycogen contents characterized by hyperglycemia were associated with GK mutation in MODY-2 subjects. The significant delay in insulin secretion in response to glucose change and reduced hepatic glucose uptake and glycogen storage in type 2 diabetes patients is closely correlated with reduced glucokinase expression in the pancreas and liver, which is considered as the underlying cause of disease. ${ }^{9,10}$ Enlightened by Matschinsky, pharmaceutical companies have launched a major effort to develop glucokinase activators(GKAs) for the treatment of diabetes in the last two decades. The lack of success in two decades' discovery of GKA was primarily due to the insufficient understanding of glucokinase as a glucose sensor, and developing GKA as a glucose processor in the glucose control. ${ }^{11}$ A new hope of GKA for T2D treatment was illustrated with a phase 2 trial success of dorzagliatin. ${ }^{12}$

Dorzagliatin is a novel, dual-acting, full glucokinase activator that activates both the pancreatic and hepatic glucokinase to improve the glucose sensitivity in T2D patients. To date, dorzagliatin has completed multiple phase 1 trials ${ }^{13-15}$ and a phase 2 trial ${ }^{16}$ both in China and the United States, and glucose sensitivity was assessed in different studies. After dorzagliatin treatment for 5.5 days in type 2 diabetes patients, the $C$-peptide secretion $T_{\max }$ was 30 minutes earlier than placebo (NCT02077452), and after dorzagliatin treatment for 28 days, early-phase insulin secretion index $(\Delta \mathrm{C} 30 / \Delta \mathrm{G} 30)$ and homeostasis model assessment 2- $\beta$ (HOMA2- $\beta$ ) increased significantly from baseline (NCT02386982). ${ }^{14}$ Dorzagliatin monotherapy for 12 weeks showed that the improvement on disposition index was observed and maintained even at a week after drug withdrawal. ${ }^{16}$ This trial (SEED) is the phase 3 trial of dorzagliatin in drug-naïve type 2 diabetes patients for a 24-week double-blind treatment and followed by a 28-week open-label treatment. Here we report the efficacy and safety outcomes of this trial.

\section{Results}

\section{Patient Demographics and Clinical Characteristics}

Patients were recruited from July 18, 2017 to February 28, 2019, with the last patient visit conducted on March 6, 2020. Of the 975 patients screened, 650 patients entered the placebo run-in period, 463 eligible patients were randomly assigned to either the dorzagliatin $75 \mathrm{mg}$ twice a day group $(n=310)$ or the placebo group ( $n=153$; Fig. 1b), and 457 patients took at least one dose of study medication and had glycated hemoglobin $(\mathrm{HbA} 1 \mathrm{c})$ measurements at baseline and at least one post-baseline timepoint. Among all patients who underwent randomization, 401 patients (86.6\%) $(281$ [90.6\%] in the dorzagliatin group and $120[78.4 \%]$ in the placebo group) completed 24 weeks of double-blind treatment and entered into 28 
weeks of open-label study. Subsequently, 351 patients (75.8\%) completed 52 weeks of treatment (246 [79.4\%] in the dorzagliatin group and 105 [68.6\%] in the placebo group) (Fig. 1b). 12 patients

discontinued for not completing on-site visit due to the influence of coronavirus pandemic in the extended 28-week open-label treatment period. Baseline characteristics were similar between the two groups (Table 1).

\section{Efficacy Outcomes}

The glycated hemoglobin level at week 24 was reduced from baseline by 1.07 percentage points in the dorzagliatin group, whereas the level decreased by 0.50 percentage points in the placebo group (estimated treatment difference, -0.57 percentage points; $95 \% \mathrm{Cl},-0.79$ to $-0.36 ; \mathrm{P}<0.001$ ) (Table 2 and Fig. 2a). This demonstrated the superiority of dorzagliatin to placebo in decreasing HbA1c. The homeostasis model assessment $2-\beta$ was consistently improved at week 24 in the dorzagliatin group compared with the placebo group ( 2.56 with dorzagliatin vs. -0.72 with placebo; estimated treatment difference, $3.28 ; 95 \% \mathrm{Cl}$, 0.44 to $6.11, P<0.05$ ) (Table 2 and Fig. $2 b$ ).

The time to first achieve a HbA1c level of less than $7.0 \%$ in the dorzagliatin group was shorter than that for the placebo group over the 24-week treatment period (Fig. 2c). The effect of lowering HbA1c levels in the dorzagliatin group started at week 4, reached the maximum at week 12 (Supplementary Table 7), and a significantly greater reduction compared to placebo maintained over 24-week treatment period. Moreover, the effect of reducing the HbA1c level in the dorzagliatin group sustained from week 24 to week 52 (open-label treatment), with reduction in $\mathrm{HbA} 1 \mathrm{c}$ level from baseline of $1.15 \%$ and $1.11 \%$ at week 24 and week 52 respectively. The decrease of the $\mathrm{HbA1c}$ level was also observed in the placebo group after switching to dorzagliatin treatment at week 24 to week 52 (Fig. 2d).

Results showed that $42.2 \%$ of the patients in the dorzagliatin group, as compared with $17.3 \%$ in the placebo group at week 24 , achieved a homeostatic control rate (a HbA1c level of less than $7.0 \%$ without hypoglycemia) for the full-analysis set (odds ratio, $4.10 ; 95 \% \mathrm{Cl}, 2.46$ to $6.85, \mathrm{P}<0.001$ ). The similar percentage of patients $(40.5 \%)$ was achieved in the dorzagliatin group since week 8 , much higher than that in the placebo group (10.0\%) and sustained till week 24 (Fig. 2e and Supplementary Table 6). In subgroup analyses, for patients with baseline $\mathrm{HbA} 1 \mathrm{c}$ levels less than or equal to $8.0 \%$, the homeostatic control rate was higher in the dorzagliatin group compared with the placebo from week $8(63.6 \% \mathrm{vs}$. $18.6 \%$ ) to week 24 (58.7\% vs. 30.5\%; odds ratio, 3.60; $95 \% \mathrm{Cl}, 1.81$ to $7.14, \mathrm{P}<0.001)$ (Fig. 2f).

The effects of dorzagliatin in reducing 2-hour postprandial glucose and fasting plasma glucose levels at week 24 compared to placebo were also shown (Table 2). At week 24, a greater decrease in the 2-hour postprandial glucose level was observed with dorzagliatin than with placebo ( $-50.9 \mathrm{mg}$ per deciliter vs. $-9.0 \mathrm{mg}$ per deciliter; estimated treatment difference, $-41.94 \mathrm{mg}$ per deciliter; $95 \% \mathrm{Cl},-57.06$ to -26.82 , $\mathrm{P}<0.001)$. The fasting plasma glucose level was reduced more in the dorzagliatin group than that in the placebo group at week 24 (-10.4 mg per deciliter vs. $-4.7 \mathrm{mg}$ per deciliter; estimated treatment difference, $-5.94 \mathrm{mg}$ per deciliter; $95 \% \mathrm{Cl},-13.68$ to $1.98, \mathrm{P}=0.141$ ) (Table 2). 


\section{Safety Outcomes}

During the 24-week double-blind treatment period, at least one adverse event(AE) was reported in 240 out of 310 patients $(77.4 \%)$ in the dorzagliatin group and 103 out of 153 patients $(67.3 \%)$ in the placebo

group (Table 3). Most of the adverse events were mild in severity, resolved, and were considered by investigators to be unrelated to dorzagliatin or placebo. No severe hypoglycemia events and drug related serious adverse events. There was no clustering of serious adverse events (Supplementary Table 11). No serious adverse events were considered as related to dorzagliatin by investigators. Adverse events that occurred in at least $5 \%$ of the patients in either treatment group and were more frequently reported in the dorzagliatin group than the placebo group were listed in Table 3. There was no significant difference in the type and severity of adverse events in the two groups. The incidents of drug related AEs judged by investigators were low. During the open-label treatment period, both adverse events and serious adverse events remain the same in type and incidence rate as that had occurred in the double-blind treatment period. No death was reported in the trial. During the double-blind treatment period, BMI decrease in both groups were observed, with no statistical difference between the two groups. There was no statistical difference in blood pressure and vital signs between the two groups (Table 4).

Clinically significant hypoglycemia (blood glucose level $<54 \mathrm{mg}$ per deciliter) was reported in $1(0.3 \%)$ of 310 patients in the dorzagliatin group during the 24-week double-blind period and 1 of 153 patients in the placebo group during the open-label treatment period (Table 3 and Supplementary Table 10). No severe hypoglycemia was reported in the trial.

\section{Discussion}

Dorzagliatin, as a glucose sensitizer, was the first glucokinase activator that demonstrated its effects on the improvement of glucose sensitivity with good glycemic control in type 2 diabetes patients in a 12week phase 2 study, which has now completed phase 3 registration trial globally. The primary goal of SEED study was to evaluate its efficacy and safety over placebo in 24-week double-blind placebocontrolled treatment, and safety profiles (efficacy second to that) during a 28-week open-label treatment, in which subjects in both placebo and dorzagliatin-treated groups were all treated with active drug dorzagliatin. The results from this phase 3 trial (SEED) in drug-naïve type 2 diabetes patients showed the improvement of glycemic control in 24-week double-blind treatment and the effects sustained through 52 weeks. Dorzagliatin showed a fast on-set action with the HbA1c, 2-hour postprandial glucose and fasting plasma glucose levels all decreased at the first visit after 4 weeks of dorzagliatin treatment. The $\mathrm{HbA} 1 \mathrm{c}$ level reached the maximal decreases at 12 weeks, and sustained during the doubleblind treatment (Supplementary Table 7). There are 129 (42.2\% out of 307) patients in the dorzagliatin group who achieved the homeostatic control rate (a HbA1c level of less than $7.0 \%$ and without hypoglycemia) at week 24 (Fig. 2e and Supplementary Table 6). $\beta$-cell function assessed using HOMA2- $\beta$, was significantly improved in the dorzagliatin group than placebo group at week 24 . We reasoned that the fast onset and sustained glycemic control could be a result from the improvement on $\beta$-cell function with dorzagliatin through improved early-phase insulin release in type 2 diabetes patients ${ }^{14,16}$ by 
optimizing glucose sensitivity in pancreatic $\beta$-cells. A much improved glycemic control was observed in the subgroup population of patients with baseline $\mathrm{HbA} 1 \mathrm{c}$ level of no more than $8.0 \%$; a higher percentage of patients achieved the HbA1c target (less than 7.0\%) and reached within a shorter period of time (Fig. $2 \mathrm{f}$ and Table 2) in the subgroup, with the response rate of $59.5 \%$ in the subgroup (vs. $42.5 \%$ in the overall population), and the median first time to reach HbA1c target of 4.7 weeks (vs. 12.1 weeks in the overall population). Similar effects on $\mathrm{HbA1c}$ were observed in the placebo group from week 24 to week 52, which has lower $\mathrm{HbA} 1 \mathrm{c}$ baseline before switching to dorzagliatin treatment (Fig. 2d). In addition, results showed that the efficacy sustained in the dorzagliatin group during the 28-week open-label treatment period (HbA1c of $1.15 \%$ vs $1.11 \%$ at week 24 and 52 using descriptive data, Supplementary Table 7). These data could offer a potential new treatment strategy to type 2 diabetes patients using dorzagliatin as a first-line therapy.

We found that dorzagliatin was well-tolerated and had a good safety profile during the entire 52-week treatment period, with no occurrence of drug-related serious adverse events or severe hypoglycemia in the dorzagliatin group. The incidence of adverse events was similar between the dorzagliatin group and the placebo group. Clinically significant hypoglycemia occurred in two patients $(0.3 \%)$ treated with dorzagliatin and were mild in nature. The homeostatic control rate was significantly improved with dorzagliatin (Fig. 2e), which is consistent with the improvement of glucose sensitivity in a-cells indicated by the improved fasting glucagon observed in type 2 diabetes rat. ${ }^{17}$

As the drug-naïve, newly-diagnosed diabetes patients in China, the characteristics of the patients who enrolled into the SEED study were observed at baseline. Although the duration of disease was reported as about 1 year, the glycemic control was poorly managed, with $\mathrm{HbA} 1 \mathrm{c}$ level about $8.3 \%$, accompanied by high 2-hour postprandial glucose about $320.6 \mathrm{mg} / \mathrm{dl}$ and the fasting $C$ peptide about $1.7 \mathrm{ng} / \mathrm{mL}$ (Table 1). The majority of subjects were not obese and body-mass index was about $25 \mathrm{~kg} / \mathrm{m}^{2}$. Lifestyle changes showed benefits in these patients. Engaging education, self-monitoring, and dietary advices from investigators during the trial contributes to body weight reduction and glycemic control during the trial. A similar reduction of $\mathrm{HbA} 1 \mathrm{c}$ was reported in the placebo group in the trials conducted in China and the strict diet and exercise control was recommended by the American Diabetes Association (ADA) and Chinese Diabetes Society (CDS) diabetes management guidelines. However, the significant improvement of $\beta$-cell function in the dorzagliatin group was observed while the deterioration of that was found in the placebo group, suggesting the benefits of improved glucose sensitivity by dorzagliatin treatment. Dorzagliatin clearly brought in additional benefits on the glycemic control in these patients, while more importantly improved their $\beta$-cell function over the placebo group (Fig. 2a and 2b).

For safety profiles, the baseline status of subjects in the SEED study reflected the characteristics of newly diagnosed Chinese patients with type 2 diabetes that have high rate of diabetes complications and concomitant diseases, such as about $55 \%$ of patients with dyslipidemia related diseases, about $45 \%$ with liver and biliary system diseases, about $39 \%$ with vascular and lymphatic diseases, about $30 \%$ with kidney and urinary system diseases and with the high level of triglyceride(about $2.0 \mathrm{mmol} / \mathrm{L}$ at baseline) at screening(Table 1). In the dorzagliatin group, no weight gain, no increased blood pressure and no 
abnormal changes of renal function in mean values were observed during 52 weeks of dorzagliatin treatment. For the several indicators of concern to other GKA molecules, the incidence of adverse events of hyperlipidemia was similar in two groups, with $11.9 \%$ in the dorzagliatin group and $10.5 \%$ in the placebo group and there was no hyperlipidemia adverse event that related to the study drug judged by investigators in the dorzagliatin group (Table 3), which suggested that long-term use of the dorzagliatin does not increase the risk of dyslipidemia. Results showed similar trend for liver function indicators that there was no drug-induced liver injury (DILI) event reported in the study. The incidence of adverse events of abnormal hepatic function was $5.8 \%$ in the dorzagliatin group and $3.9 \%$ in the placebo group, and 2 cases in the placebo group and 2 cases in the dorzagliatin group were reported as related to the study drug judged by investigators. The results also suggested that long-term use of the dorzagliatin does not increase the risk of abnormal liver function.

The limitation of this study is that the SEED trial was only conducted in Chinese drug-naive type 2 diabetes patients, and thus the efficacy and safety results should be investigated in other populations who are different in terms of disease etiology, environmental factors, and the treatment regimens. To address this, dorzagliatin has been evaluated in several clinical trials conducted in the United States. Three phase 1 drug-drug interaction trials with metformin, dipeptidyl peptidase 4 inhibitor (sitagliptin) and sodium-glucose co-transporter-2 inhibitor (empagliflozin) (NCT03790839 and NCT03790787) were completed with synergistic effects in the glycemic control to support future investigation in non-Chinese population. A 24-week double-blind and extended 28-week open-label treatment phase 3 trial (NCT03141073) in Chinese patients with insufficient glycemic control by metformin alone was recently completed in China that further supported the synergy of dorzagliatin with metformin in the glycemic control, with a significant reduction of postprandial glucose and the improvement of $\beta$-cell function and insulin sensitivity. In SEED study, the 52-week treatment period in the dorzagliatin group consisted of a 24-week double-blind treatment and a 28-week open-label treatment period. Further studies will be conducted to validate the longer-term efficacy and safety of dorzagliatin.

In conclusion, dorzagliatin monotherapy significantly improved the glycemic control starting at the week 4 and maintained till week 24, and the treatment was safe and well-tolerated over the 52 weeks. In addition, the glycemic control sustained over 52 weeks in the dorzagliatin group after the extended 28week open-label treatment.

\section{Methods}

\section{Trial Design and Oversight}

We conducted this phase 3 trial (SEED) from July 18, 2017 to March 6, 2020, at 40 sites in China, in Chinese drug-naïve type 2 diabetes patients. The SEED trial had a 2-week screening period, a 4-week single-blind placebo run-in period, and then eligible patients were randomized at a 2:1 ratio into the dorzagliatin and placebo group in a 24-week double-blind, placebo-controlled treatment period, followed 
by an extended 28-week open-label treatment period with all patients receiving dorzagliatin orally, and a 1-week follow-up period without treatment (Fig. 1a).

The trial was conducted in accordance with the principles of Declaration of Helsinki, Good Clinical Practice guidelines, and laws and regulations in China. The study protocol was amended once during study enrollment, and the important changes to methods after trial commencement and reasons were summarized in the supplemental material. The trial protocol and amendments were approved by the local ethics committees of all study sites. Written informed consent was obtained from all patients before the initiation of any trial-related procedures. The trial was also conducted in accordance with the Chinese Diabetes Society guidelines, which requires physicians to educate and strictly enforce improved exercise and dietary control, as well as self-monitoring of blood glucose (at least 2 times per week), in treating type 2 diabetes patients.

\section{Patients}

Patients were recruited at the study site by investigators. Patients were required to sign the informed consent form before enrollment. After providing written informed consent, patients were screened for eligibility. The individuals ( 18 to 75 years of age) were eligible for inclusion in the trial if they met the following criteria: diagnosed with type 2 diabetes, on diet and exercise interventions for at least 3 months and had no antidiabetic therapy before screening, had $\mathrm{HbA1c}$ levels between 7.5 and $11.0 \%$, and had a body-mass index between 18.5 and $35.0 \mathrm{~kg}$ per square meters at screening.

Individuals were excluded if they had severe hypoglycemia without cause within 3 months, or frequent hypoglycemia occurred no less than 3 times within 1 month before screening, fasting C-peptide level of less than $1.0 \mathrm{ng}$ per milliliter at screening, a history of type 1 diabetes. Other exclusion criteria were the major cardio-cerebrovascular diseases within 6 months before screening; unstable or rapidly progressive kidney disease; active liver diseases; psychiatric disease; any type of malignancy; an alanine or aspartate transaminase level greater than 2.5 times of the upper limit of the normal range, or serum total bilirubin level greater than 1.5 times of the upper limit of the normal range. Complete lists of inclusion, exclusion, and randomization criteria are provided in the protocol as a supplemental material.

\section{Trial Procedures}

After screening, eligible patients entered a 4-week, single-blind placebo run-in period. At week 3 of run-in period, patients were reevaluated before randomization to confirm the eligibility. Eligibility criteria included $\mathrm{HbA} 1 \mathrm{c}$ levels between 7.5 and $10.0 \%$, and fasting plasma glucose levels between 7.0 and 13.3 $\mathrm{mmol}$ per liter at randomization. Diet and exercise counseling was provided during the entire experiment.

Eligible patients were randomly assigned, in a 2:1 ratio, to receive dorzagliatin or placebo for 24 weeks, on a background of a diet and exercise regimen. Randomization and drug dispense were performed with an interactive web response system (IWRS) (Balance, MedData Solution). The random allocation sequences were generated by the IWRS. A stratified randomization method with the permuted block randomization 
algorithm was used. The blocks were dynamically allocated to each site and stratum from the randomization list. A unique identification number (drug box serial number) was provided by the drug packing vendor and marked on the label of drug box. By central randomization, the randomization codes were assigned to subjects who meet randomization criteria by IWRS system based on randomization factors (baseline $\mathrm{HbA} 1 \mathrm{c}$ level $\leq 8.5 \%$ or $>8.5 \%$ ) and the block size. Then, the randomization codes along with the corresponding drug box numbers were provided to subjects who meet the randomization criteria at each visit.

Investigators enrolled participants and used IWRS to assign participants to interventions. Double-blind method was used in the study. The random allocation sequences were concealed from patients, investigators and all other study members. The placebo tablets have the size, color, odor, and appearance same as the investigational drug. Blinding was maintained until the end of week 24 . All doses of dorzagliatin $75 \mathrm{mg}$ tablets and placebo tablets were administered orally twice daily, as single tablet.

All the patients who completed a 24-week double-blind treatment (dorzagliatin or placebo), received dorzagliatin treatment for a 28-week open-label period, during which patients assigned to dorzagliatin continued their treatment unchanged, whereas those assigned to placebo were switched from the placebo to dorzagliatin. After the 52-week treatment, all patients were observed for 1 week for safety evaluation.

\section{Endpoints}

The primary efficacy endpoint was the change from baseline in the HbA1c level at week 24. Key secondary efficacy endpoints included the changes from baseline in 2-hour postprandial glucose, fasting plasma glucose levels at week 24, and $\mathrm{HbA} 1 \mathrm{c}$ level at each visit (except week 24); the percentage of patients who reached a $\mathrm{HbA} 1 \mathrm{c}$ level of less than $7.0 \%$ at week 24 . Additional efficacy endpoints included homeostatic control rate (a HbA1c level of less than $7.0 \%$ and without hypoglycemia), as well as the change from baseline in the homeostasis model assessment 2- $\beta$ (an index for $\beta$-cell function). A full list of other efficacy endpoints is provided in the statistical analysis plan as the supplemental material.

Adverse events, serious adverse events were assessed throughout the trial (definitions are provided in the protocol as a supplemental material). Hypoglycemic episodes were classified according to the American Diabetes Association definitions (see the protocol provided as a supplemental material). Vital signs and clinical laboratory test results were assessed, and physical examinations were performed.

\section{Statistical Analyses}

We hypothesized that dorzagliatin would show superiority to placebo in the decrease of HbA1c level in patients after 24-week treatment. For the primary endpoint of the change from baseline in the HbA1c level at week 24 , we calculated that a total sample size of 450 patients would provide the trial with $91.4 \%$ power to detect a difference of 0.4 percentage points between the dorzagliatin group and the placebo group in a 2:1 ratio of allocation at a 2-sided significance level of 0.05 , assuming a standard deviation 
(SD) of 1.2 percentage points. The full analysis set included all randomized patients, who took at least one dose of study drugs and had at least one post-treatment measurement of the primary endpoint during double-blind treatment period. The safety set included all randomized patients, who took at least one dose of study drugs. Since this trial had only one confirmatory hypothesis test, i.e. the test of null hypothesis of no difference in the primary endpoint between dorzagliatin and placebo, there was no adjustment for multiplicity.

The primary analysis method for the primary efficacy endpoint was in the full analysis set using a mixed model for repeated measures without missing value imputation, which included treatment group, scheduled visit, interaction of treatment group with scheduled visit, pooled site and $\mathrm{HbA} 1 \mathrm{c}$ baseline value as fixed effects. In the mixed model for repeated measures, data collected after initiation of prohibited antidiabetic medications (the amount and time of taking were reviewed in blind-data-review meeting and open-data-review meeting) were handled as missing data. The least-squares means of each treatment group, the estimated treatment difference between dorzagliatin and placebo and its $95 \%$ confidence interval $(\mathrm{Cl})$ were calculated. A sensitivity analysis was performed with the primary efficacy endpoint in the full analysis set using an analysis of covariance model with factors of treatment group, pooled site and interaction of treatment group with pooled site (interaction effect removed if not significant on a 2sided alpha level of 0.1 ), and a covariate of $\mathrm{HbA1c}$ baseline value. In the analysis of covariance model, missing values were imputed using the method of last observation carried forward, which was applied after excluding data collected after initiation of prohibited antidiabetic medications. Another sensitivity analysis was performed with the primary efficacy endpoint using the same mixed model for repeated measures in the per-protocol set.

The secondary efficacy endpoints including changes from baseline in 2-hour postprandial glucose, and fasting plasma glucose levels at week 24 were assessed using the same mixed model for repeated measures for the primary endpoint. Another secondary efficacy endpoint of the percentage of patients who reached a $\mathrm{HbA} 1 \mathrm{c}$ level of less than $7.0 \%$ and additional efficacy endpoint of the homeostatic control rate were estimated based on the data imputed by last observation carried forward approach in the full analysis set. Odds ratio and $95 \%$ confidence interval between two treatment groups were estimated using the logistic regression model with categorical independent variables of treatment group, pooled site and interaction of treatment group with pooled site (interaction effect removed from the model if not significant at a 2-sided alpha level of 0.1 ), and a continuous independent variable of baseline $\mathrm{HbA} 1 \mathrm{c}$ level. For other efficacy endpoints, such as the change from baseline in the homeostasis model assessment $2-\beta$ were analyzed using the same analysis of covariance model as described above.

The post-hoc subgroup analyses were carried out with the same statistical methods as used for primary efficacy outcome. Patients were stratified into subgroups according to their $\mathrm{HbA1c}$ level at baseline (less than or equal to $8.0 \%$ vs greater than $8.0 \%$ ). The analysis of covariance model was used to investigate the treatment effect on homeostasis model assessment 2- $\beta$. Kaplan-Meier method was employed to analyze the time to first reach target response (a HbA1c level of less than $7.0 \%)$ over 24 weeks. 
The incidence of treatment-emergent adverse events that occurred between the first intake of double-blind study medications and the 7th day after the last dose of study medications was summarized by treatment group and compared between two treatment groups. The incidence of hypoglycemic events was also summarized by treatment group and compared between treatment groups, both patients that spontaneously reported low glucose events and low glucose events reported at on-site visit were counted as hypoglycemic events. Additional details regarding the statistical analysis are provided in the statistical analysis plan as a supplemental material.

\section{Declarations}

Reporting Summary. Further information on research design is available in the Nature Research Reporting Summary linked to this article.

\section{Data availability}

We prefer not to share the data collected for our study, including individual patient data and a data dictionary defining each field in the data set.

\section{Code availability}

Commercially available software (SAS version 9.4, SAS Institute) was used for analyses.

\section{Acknowledgements}

We thank Jing Chen, Ph.D., for medical writing and editing support, funded by Hua Medicine; Hui Wang, Yue Zhang, and Yuan Zhang (Hua Medicine), for assistance with the efficacy data; Aihua Wang (Hua Medicine), for assistance with the safety data; Xiang Liu (Hua Medicine), for assistance with the statistical analyses; and Gary Yu and Fuxing Tang, who reviewed an earlier version of the manuscript on behalf of Hua Medicine. The study is funded by Hua Medicine, and in part, by grants from the National Major Scientific and Technological Special Project for Significant New Drugs Development (2014ZX09101002004, 2018ZX09711002-012-001), Shanghai Science and Technology Innovation Action Project (14431908300, 15XD1520500, 17DZ1910200, 19431905200), Shanghai Pudong District Science and Technology Innovation Action Project (PKJ2014-S06), Shanghai Municipal Commission of Economy and Informatization Innovation Action Project (XC-ZXSJ-01-2015-02, 18XI-18).

\section{Author contributions}

Dalong Zhu led the design, conduct and analysis of the clinical study, as well as the development of the manuscript. These authors contributed equally: Dalong Zhu, Xiaoying Li. The trial was designed and overseen by representatives of the trial sponsor (Hua Medicine) with input from selected site investigators. The site investigators collected the data, and the sponsor performed the data analyses. All the authors interpreted the data, and vouch for the accuracy and completeness of the data and the fidelity of the trial to the protocol. The first author wrote the first draft of the manuscript with assistance from an 
independent medical writer funded by the sponsor. The manuscript was subsequently revised and approved by all the authors, who agreed to submit the manuscript for publication.

\section{Competing interests}

The authors declare no competing interests.

\section{References}

1. American Diabetes Association. Introduction: Standards of Medical Care in Diabetes-2020. Diabetes Care. 2020 Jan;43(Suppl 1):S1-S2.

2. RISE Consortium. Lack of durable improvements in $\beta$-cell function following withdrawal of pharmacological interventions in adults with impaired glucose tolerance or recently diagnosed type 2 diabetes. Diabetes Care. 2019 Sep 1;42(9):1742-51.

3. American Diabetes Association. 6. Glycemic Targets: Standards of Medical Care in Diabetes-2020. Diabetes Care. 2020 Jan;43(Suppl 1):S66.

4. Matschinsky FM, Ellerman JE. Metabolism of glucose in the islets of Langerhans. Journal of Biological Chemistry. 1968 May 25;243(10):2730-6.

5. Matschinsky FM, Wilson DF. The central role of glucokinase in glucose homeostasis: A perspective 50 years after demonstrating the presence of the enzyme in islets of Langerhans. Frontiers in physiology. 2019 Mar 6;10:148.

6. Matschinsky FM, et al. Research and development of glucokinase activators for diabetes therapy: theoretical and practical aspects. Diabetes-Perspectives in Drug Therapy. 2011:357-401.

7. Singh Grewal A, Singh Sekhon B, Lather V. Recent updates on glucokinase activators for the treatment of type 2 diabetes mellitus. Mini Reviews in Medicinal Chemistry. 2014 Jun 1;14(7):585602.

8. Matschinsky FM. Assessing the potential of glucokinase activators in diabetes therapy. Nature reviews Drug discovery. 2009 May;8(5):399-416.

9. Froguel P, et al. Familial hyperglycemia due to mutations in glucokinase--definition of a subtype of diabetes mellitus. New England Journal of Medicine. 1993 Mar 11;328(10):697-702.

10. Haeusler RA, et al. Decreased expression of hepatic glucokinase in type 2 diabetes. Molecular metabolism. 2015 Mar 1;4(3):222-6.

11. Matschinsky FM. GKAs for diabetes therapy: why no clinically useful drug after two decades of trying?. Trends in pharmacological sciences. 2013 Feb 1;34(2):90-9.

12. Scheen AJ. New hope for glucokinase activators in type 2 diabetes?. The Lancet Diabetes \& Endocrinology. 2018 Aug 1;6(8):591-3.

13. Xu H, et al. Safety, tolerability, pharmacokinetics, and pharmacodynamics of novel glucokinase activator HMS5552: results from a first-in-human single ascending dose study. Drug design, development and therapy. 2016;10:1619. 
14. Zhu XX, et al. Dorzagliatin (HMS5552), a novel dual-acting glucokinase activator, improves glycaemic control and pancreatic $\beta$-cell function in patients with type 2 diabetes: A 28-day treatment study using biomarker-guided patient selection. Diabetes, Obesity and Metabolism. 2018 Sep;20(9):2113-20.

15. Chen L, Zhao G, Ren S, Zhang Y, Du D. No drug-drug interaction between dorzagliatin and metformin in type 2 diabetes patients. Diabetes 2018 Jul; 67(Supplement 1): -

16. Zhu D, et al. Dorzagliatin monotherapy in Chinese patients with type 2 diabetes: a dose-ranging, randomised, double-blind, placebo-controlled, phase 2 study. The Lancet Diabetes \& Endocrinology. 2018 Aug 1;6(8):627-36.

17. Wang P, et al. Effects of a novel glucokinase activator, HMS5552, on glucose metabolism in a rat model of type 2 diabetes mellitus. Journal of diabetes research. 2017 Jan 16;2017.

18. Chung WK, et al. Precision medicine in diabetes: a consensus report from the American Diabetes Association (ADA) and the European Association for the Study of Diabetes (EASD). Diabetologia. 2020 Sep;63(9):1671-93.

\section{Tables}


Table 1. Demographic and Baseline Characteristics*

\begin{tabular}{|c|c|c|}
\hline $\begin{array}{l}\text { Dorzagliatin } \\
(\mathrm{N}=310)\end{array}$ & $\begin{array}{l}\text { Placebo } \\
(\mathrm{N}=153)\end{array}$ & \\
\hline Age - yr & $53.2 \pm 9.60$ & $53.5 \pm 10.02$ \\
\hline Male sex - n. (\%) & $200(64.5)$ & $101(66.0)$ \\
\hline Duration of disease - mth & $12.11 \pm 8.98$ & $10.70 \pm 7.96$ \\
\hline Body weight - kg & $69.75 \pm 10.759$ & $68.28 \pm 9.837$ \\
\hline Body mass index ${ }^{\S}-\mathrm{kg} / \mathrm{m}^{2}$ & $25.60 \pm 2.94$ & $25.32 \pm 2.75$ \\
\hline \multicolumn{3}{|l|}{ Blood pressure - mm Hg } \\
\hline Systolic & $125.3 \pm 13.1$ & $124.0 \pm 13.6$ \\
\hline Diastolic & $79.8 \pm 8.5$ & $78.4 \pm 8.6$ \\
\hline Glycated hemoglobin - \% & $8.34 \pm 0.67$ & $8.38 \pm 0.73$ \\
\hline$\leq 8 \%-$ no. $(\%)$ & $123(39.68)$ & $59(38.56)$ \\
\hline$>8 \%-$ no. $(\%)$ & $187(60.32)$ & $94(61.44)$ \\
\hline Fasting plasma glucose - mg/dl & $176.0 \pm 32.2$ & $174.8 \pm 27.6$ \\
\hline 2-hour postprandial glucose - mg/dl & $320.6 \pm 57.1$ & $321.3 \pm 57.2$ \\
\hline Fasting C peptide - ng/mL & $1.762 \pm 0.6430$ & $1.738 \pm 0.6188$ \\
\hline HOMA2- $\beta$ & $35.14 \pm 13.983$ & $34.75 \pm 13.587$ \\
\hline Medical history - n(\%) & $291(93.9)$ & $144(94.1)$ \\
\hline Metabolic and nutritional diseases & $180(58.1)$ & $95(62.1)$ \\
\hline Hyperlipemia & $121(39.0)$ & $62(40.5)$ \\
\hline Dyslipidemia & $31(10.0)$ & $17(11.1)$ \\
\hline Hypertriglyceridemia & $12(3.9)$ & $6(3.9)$ \\
\hline Low HDL cholesterolemia & $6(1.9)$ & $4(2.6)$ \\
\hline Hypercholesterolemia & $2(0.6)$ & $4(2.6)$ \\
\hline Liver and biliary system diseases & $138(44.5)$ & $72(47.1)$ \\
\hline Vascular and lymphatic diseases & $123(39.7)$ & $60(39.2)$ \\
\hline Kidney and urinary system diseases & $93(30.0)$ & $52(34.0)$ \\
\hline Infectious and infectious diseases & $90(29.0)$ & $43(28.1)$ \\
\hline Heart organ disease & $58(18.7)$ & $24(15.7)$ \\
\hline Neurological diseases & $39(12.6)$ & $22(14.4)$ \\
\hline ALT-U/L & $23.3(12.66)$ & $22.4(11.02)$ \\
\hline AST-U/L & $20.0(7.55)$ & $20.5(8.30)$ \\
\hline TBill- umol/L & $11.4(4.21)$ & $11.7(4.26)$ \\
\hline $\mathrm{TG}-\mathrm{mmol} / \mathrm{L}$ & $1.992(1.1556)$ & $2.050(1.0948)$ \\
\hline $\mathrm{TC}-\mathrm{mmol} / \mathrm{L}$ & $4.846(0.9518)$ & $4.890(0.9012)$ \\
\hline LDL-C - mmol/L & $2.775(0.8389)$ & $2.776(0.7605)$ \\
\hline HDL-C - mmol/L & $1.190(0.2750)$ & $1.182(0.2771)$ \\
\hline eGFR + - mL/min $/ 1.73 \mathrm{~m}^{2}$ & $96.24(17.187)$ & $97.10(20.236)$ \\
\hline
\end{tabular}

* Plus-minus values are means \pm SD. $§$ Body-mass index (BMI) is the weight in kilograms divided by the square of the height in meters. SD: standard deviation $\dagger$ eGFR was calculated in 766 patients who were in the safety set population. 
Table 2. Changes in Efficacy Endpoints From Baseline to Week 24.

\begin{tabular}{|c|c|c|c|c|}
\hline End Point & $\begin{array}{l}\text { Dorzagliatin } \\
\quad(\mathrm{N}=307)\end{array}$ & $\begin{array}{l}\text { Placebo } \\
(\mathrm{N}=150)\end{array}$ & $\begin{array}{l}\text { Estimated Treatment } \\
\text { Difference, Dorzagliatin } \\
\text { vs. Placebo }(95 \% \mathrm{CI})\end{array}$ & $\begin{array}{c}\mathrm{P} \\
\text { Value }\end{array}$ \\
\hline \multicolumn{5}{|l|}{ Glycated hemoglobin $*_{-} \%$} \\
\hline LS mean change from baseline & $-1.07 \pm 0.061$ & $-0.50 \pm 0.091$ & $-0.57(-0.79,-0.36)$ & $<0.001$ \\
\hline \multicolumn{5}{|l|}{ 2-hour postprandial glucose $*_{-} \mathrm{mg} / \mathrm{dl}$} \\
\hline LS mean change from baseline & $-50.9 \pm 4.4$ & $-9.0 \pm 6.6$ & $-41.94(-57.06,-26.82)$ & $<0.001$ \\
\hline \multicolumn{5}{|l|}{ Fasting plasma glucose $*_{-} \mathrm{mg} / \mathrm{dl}$} \\
\hline LS mean change from baseline & $-10.4 \pm 2.2$ & $-4.7 \pm 3.3$ & $-5.94(-13.68,1.98)$ & $=0.141$ \\
\hline \multicolumn{5}{|c|}{ Response rate (glycated hemoglobin $<7.0 \%)^{\dagger}{ }_{-} \%$} \\
\hline $\begin{array}{l}\text { Percentage of patients achieving target } \\
\text { (Overall population) }\end{array}$ & $42.5 \%$ & $17.3 \%$ & $4.20(2.51,7.02) \|$ & $<0.001$ \\
\hline $\begin{array}{l}\text { Percentage of patients achieving target } \\
\text { (Baseline glycated hemoglobin } \leq 8 \% \\
\text { population) }\end{array}$ & $59.5 \%$ & $30.5 \%$ & $3.80(1.90,7.57)$ & $<0.001$ \\
\hline \multicolumn{5}{|l|}{ Homeostasis model assessment $2-\beta \ddagger$} \\
\hline LS mean change from baseline & $2.56 \pm 0.852$ & $-0.72 \pm 1.220$ & $3.28(0.44,6.11)$ & $<0.05$ \\
\hline
\end{tabular}

First time to reach glycated hemoglobin $<7.0 \%$ target (glycated hemoglobin control rate)- week $\dagger$

\begin{tabular}{|c|c|c|c|c|}
\hline $\begin{array}{l}\text { Median time }(95 \% \mathrm{CI}) \\
\text { (Overall population) }\end{array}$ & $\begin{array}{c}12.1(12.0 \\
16.1)\end{array}$ & NE§ & - & $<0.001$ \\
\hline Median time (95\% CI) (Baseline glycated & $4.7(4.3,8.1)$ & $24.6(16.6, \mathrm{NE})$ & - & $<0.001$ \\
\hline
\end{tabular}
hemoglobin $\leq 8 \%$ population)

* Plus-minus values are estimated LS means \pm SE. LS mean differences and corresponding 95\% confidence interval (CI) were estimated using a mixed model for repeated measures in the full-analysis set.

† Response rate was estimated in the full-analysis set, with the use of the data imputed by last observation carried forward. Estimated treatment difference is represented as the odds ratio and 95\%CI using logistic regression.

‡ Plus-minus values are estimated LS means \pm SE. LS mean differences were calculated from an analysis of covariance in the full-analysis set, with the use of the data imputed by last observation carried forward.

SE: standard error; SD: standard deviation; NA: not available; LS: least-squares; CI: confidence interval. 


\begin{tabular}{|c|c|c|}
\hline \multirow[t]{2}{*}{ Event } & $\begin{array}{l}\text { Dorzagliatin } \\
(\mathrm{N}=310)\end{array}$ & $\begin{array}{l}\text { Placebo } \\
(\mathrm{N}=153)\end{array}$ \\
\hline & No. of Patients (\%) & No. of Patients (\%) \\
\hline Any adverse events & $240(77.4)$ & $103(67.3)$ \\
\hline Any serious adverse events & $12(3.9)$ & $9(5.9)$ \\
\hline Adverse events leading to drug discontinuation\# & 0 & $2(1.3)$ \\
\hline Serious adverse events leading to discontinuation & $2(0.6)$ & $6(3.9)$ \\
\hline \multicolumn{3}{|l|}{ Adverse events in $\geq 5 \%$ of patients $\|$} \\
\hline Upper respiratory tract infection & $58(18.7)$ & $27(17.6)$ \\
\hline Hyperlipidemia & $37(11.9)$ & $16(10.5)$ \\
\hline Protein urine present & $26(8.4)$ & $7(4.6)$ \\
\hline Hepatic function abnormal & $18(5.8)$ & $6(3.9)$ \\
\hline Hypertension & $16(5.2)$ & $6(3.9)$ \\
\hline \multicolumn{3}{|l|}{ Adverse events in at least $5 \%$ of patients related to the study drug $t$} \\
\hline Upper respiratory tract infection & $3(1.0)$ & 0 \\
\hline Hyperlipidemia & $1(0.3)$ & 0 \\
\hline Protein urine present & $1(0.3)$ & $1(0.7)$ \\
\hline Hepatic function abnormal & $2(0.6)$ & $2(1.3)$ \\
\hline Hypertension & $1(0.3)$ & 0 \\
\hline \multicolumn{3}{|l|}{ Hypoglycemia } \\
\hline Severe hypoglycemia & 0 & 0 \\
\hline Clinically significant hypoglycemia (Blood glucose level $<3.0 \mathrm{mmol} / \mathrm{L}$ ) & $1(0.3)$ & 0 \\
\hline Drop-out due to hypoglycemia & 0 & 0 \\
\hline
\end{tabular}

*Adverse events and serious adverse events that occurred in the 24-week double-blind treatment period among patients in the safety population are included in the table and presented with their preferred terms in the Medical Dictionary for Regulatory Activities, version 23.0. The safety population includes all randomized patients, who took at least one dose of study drugs. Events were included if the date of onset was between the first intake of double-blind study medications and the 7th day after the last dose of study medications.

\# Serious adverse events that lead to drug discontinuation are not included.

$\|$ Adverse events in at least $5 \%$ of patients and more frequently reported in the dorzagliatin group than in the placebo group were listed in the table. Urinary tract infection was reported in $5.2 \%$ of patients in both groups and is not listed in the table.

†Adverse events in at least $5 \%$ of patients related to the study drug was defined as adverse events that were deemed by the investigators to be very likely, or probably related to the study drug or placebo. 
Table 4. Changes in Selected Labs and Vital Signs at Week 24 (Safety Set). Outcome

Dorzagliatin

$(\mathrm{N}=310)$
Placebo

$(\mathrm{N}=153)$

\begin{tabular}{lcccc}
\hline & Week 24 & Week 52 & Week 24 & Week 52 \\
Change in body weight - $\mathrm{kg}$ & $-0.14 \pm 2.239$ & $-0.34 \pm 2.521$ & $-0.76 \pm 2.382$ & $-0.41 \pm 2.326$ \\
Change in body mass index- $\mathrm{kg} / \mathrm{m}^{2}$ & $-0.052 \pm 0.8235$ & $-0.124 \pm 0.9168$ & $-0.286 \pm 0.8927$ & $-0.157 \pm 0.8619$ \\
Change in systolic blood pressure - mm Hg & $-1.34 \pm 11.474$ & $0.38 \pm 10.190$ & $-0.80 \pm 11.094$ & $0.90 \pm 11.634$ \\
Change in diastolic blood pressure- mm Hg & $-1.28 \pm 8.020$ & $-1.28 \pm 8.020$ & $-0.06 \pm 7.676$ & $-0.06 \pm 7.676$
\end{tabular}

Plus-minus values are arithmetic means \pm SD.

SD: standard deviation.

\section{Figures}


a.

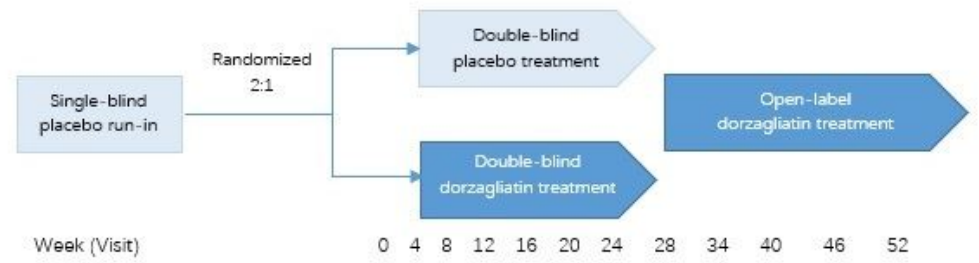

b.

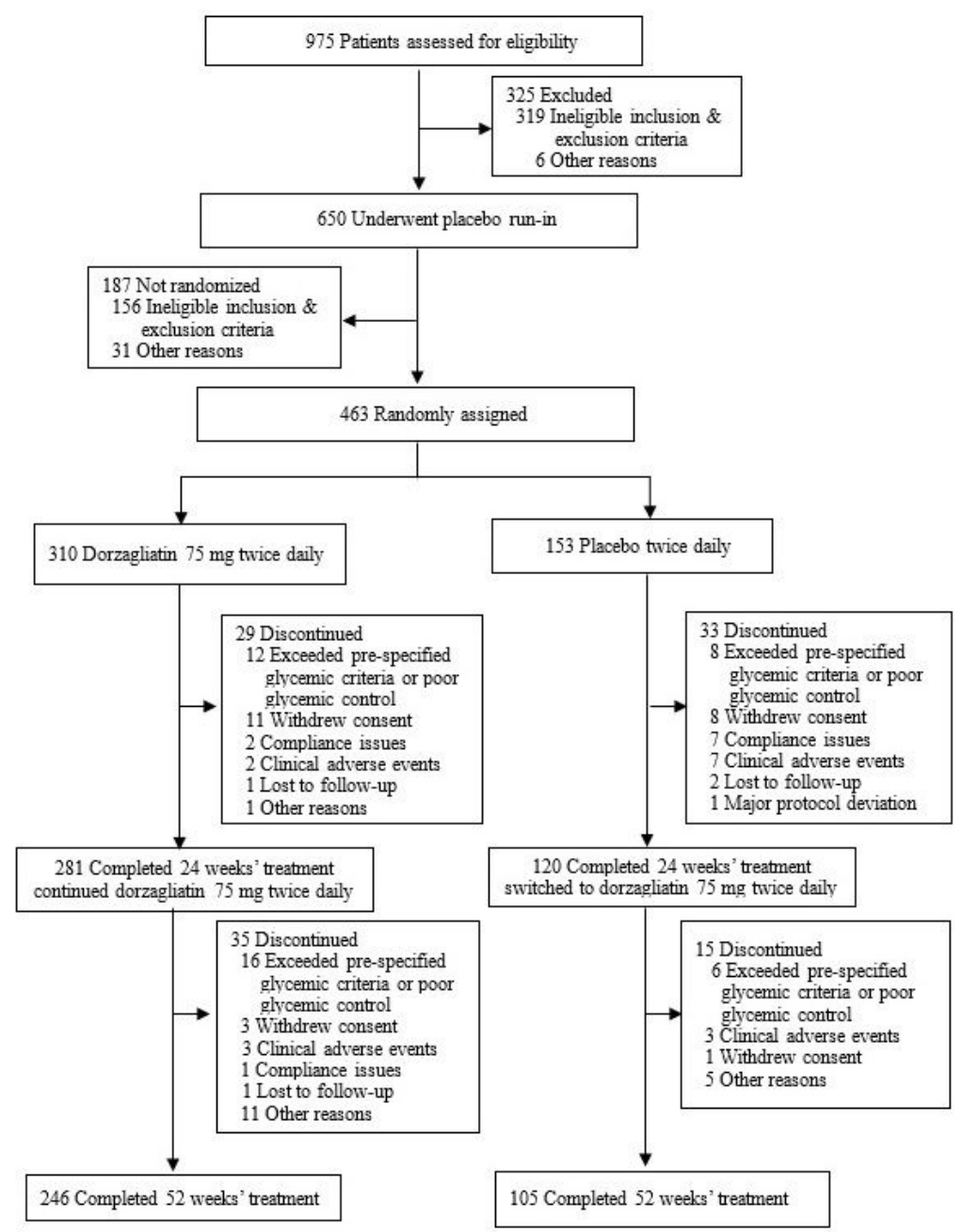

\section{Figure 1}

SEED trial design and study patient disposition. a. SEED trial design. b. SEED study patient disposition flow diagram throughout the trial. 


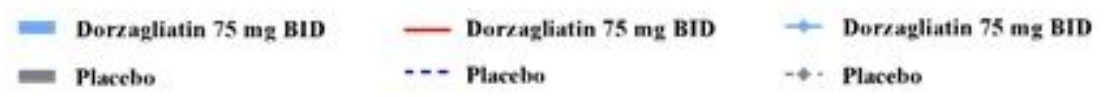

a Glycated hemoglobin change at week 24 from baseline

b HOMA2- $\beta$ change at week 24 from baseline
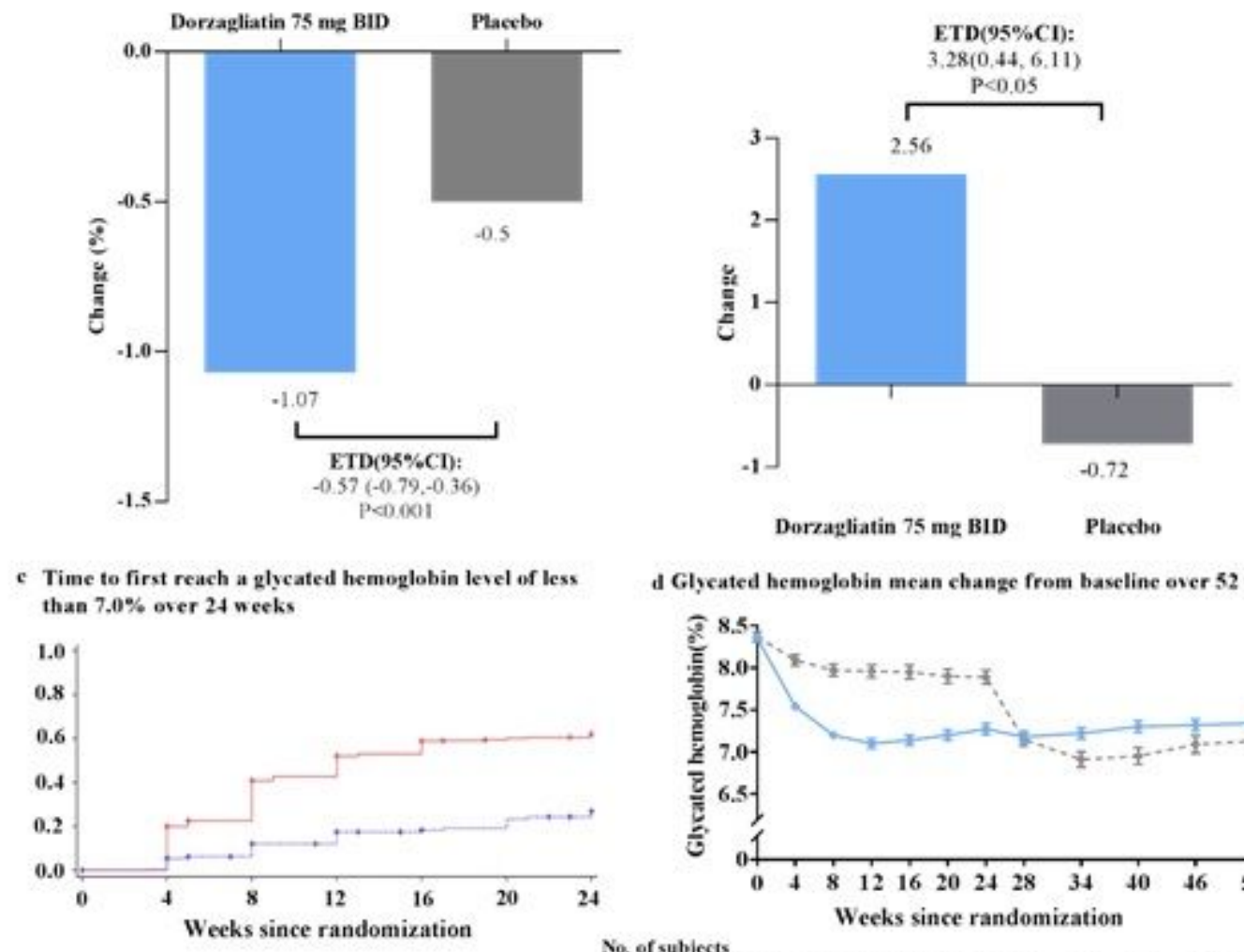

d Glycated hemoglobin mean change from baseline over 52 weeks

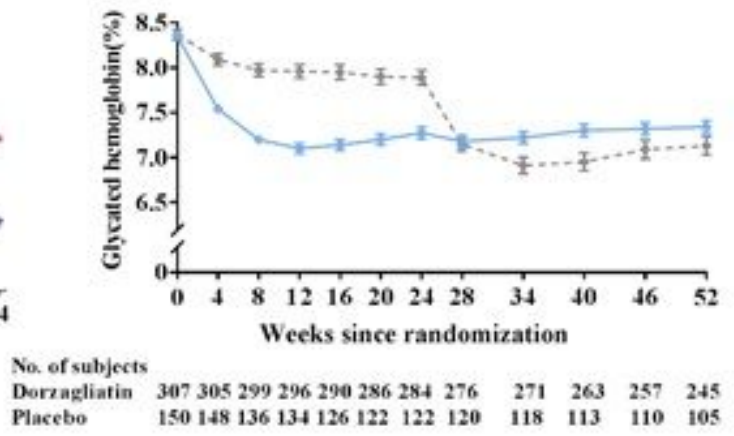

e Homeostatic control rate in the overall population

f Response rate at week 24 (baseline glycated hemoglobin $\leqslant 8 \%$ )
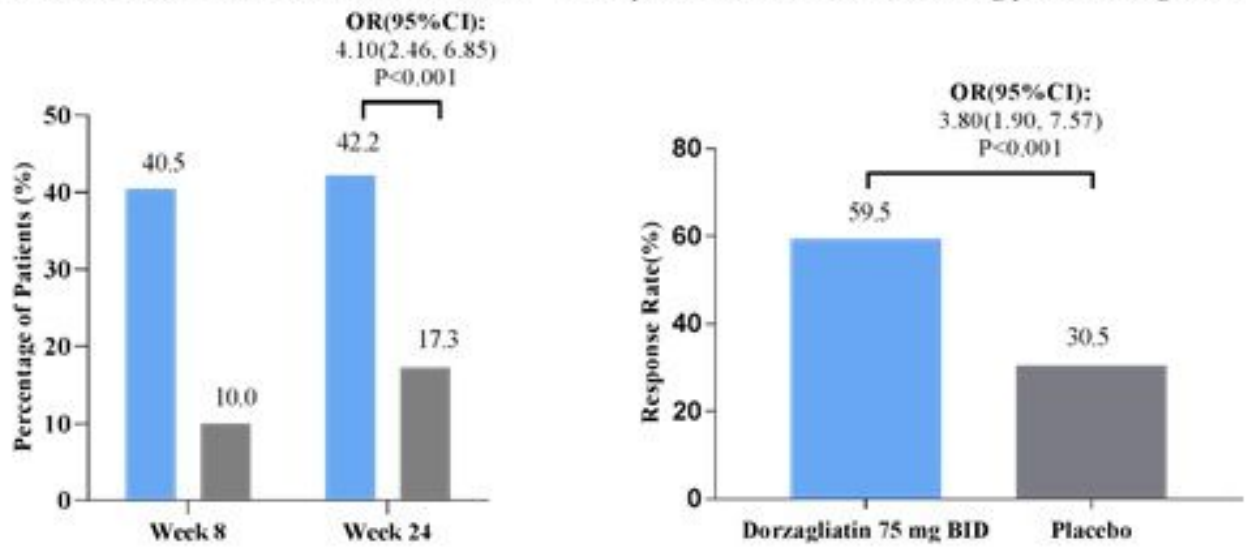

Figure 2

Efficacy Endpoints. a. It shows the least-squares (LS) mean change from baseline in the glycated hemoglobin level at week 24 in patients who received either dorzagliatin or placebo. Estimated treatment differences (ETD) and corresponding 95\% confidence interval(Cl) were estimated in the full-analysis set using a mixed model for repeated measures. $b$. It shows the LS mean change from baseline in homeostasis model assessment $2-\beta$ estimated from an analysis of covariance model. Homeostasis model assessment $2-\beta$ is an index for evaluating pancreatic $\beta$-cell function with the new homeostasis model. It is developed from homeostasis model assessment-1, including physiological indicators of 
glucose homeostasis such as liver and peripheral insulin resistance. c. It shows the time to first reach a glycated hemoglobin level of less than $7.0 \%$ over 24 weeks, which was analyzed by Kaplan-Meier method. d. It shows the arithmetic mean change in the glycated hemoglobin level over 52 weeks in patients who received either dorzagliatin or placebo. Error bars indicate standard errors. e. It shows homeostatic control rate (glycated hemoglobin of less than $7.0 \%$ and without hypoglycemia) in the overall population and patients with baseline glycated hemoglobin less than or equal to $8.0 \%$. $f$ The percentages of patients who reached glycated hemoglobin of less than $7.0 \%$ target in baseline glycated hemoglobin less than and equal to $8.0 \%$ population. SE: standard error; HR: hazard ratio.

\section{Supplementary Files}

This is a list of supplementary files associated with this preprint. Click to download.

- SEEDsupplementarytables.docx

- SEEDstudymanuscriptsupplement.pdf 